\title{
Composición proximal en morfotipos de chile Comapeño (Capsicum annuum L.) de Oaxaca y Veracruz, México, y su relación con factores climáticos
}

Proximal composition in Comapeño chili pepper morphotypes (capsicum annuum 1.) from Oaxaca and Veracruz, Mexico, and their relationship with climate factors

\author{
Gregorio Hernández-Salinas ${ }^{1}$ (D) , Alan Emilio Arenas-Lozano ${ }^{1}$ (D), \\ Mario Luna-Cavazos ${ }^{2}$ (D) , Emmanuel de Jesús Ramírez-Rivera ${ }^{1}$ (D) , Rubén Purroy-Vásquez ${ }^{1}$ (D) , \\ César Sotelo-Leyva ${ }^{4}$ iD , Rosa Isela Castillo-Zamudio ${ }^{3^{*}}$
}

\footnotetext{
${ }^{1}$ Campus Zongolica, Tecnológico Nacional de México/Instituto Tecnológico Superior de Zongolica, Km 4 Carretera a la Compañía S/N,, Tepetitlanapa, Zongolica, Veracruz, 95005, México.

${ }^{2}$ Colegio de Postgraduados (CP)-Campus Montecillo, Km 36.5 Carretera México-Texcoco, Montecillo, Estado de México, 56230, México. ${ }^{3}$ CP-Campus Veracruz, Km 88.5 Carretera Federal Xalapa-Veracruz, Veracruz, 91690, México.

${ }^{4}$ Facultad de Ciencias Químico-Biológicas, Universidad Autónoma de Guerrero, Av. Lázaro Cárdenas SN, Cd. Universitaria Sur, Chilpancingo, Guerrero, México.
}

*Autor de correspondencia: rosychely@colpos.mx

Fecha de recepción:

27 de octubre de 2020

Fecha de aceptación:

14 de mayo de 2021

Disponible en línea:

24 de septiembre de 2021

Este es un artículo en acceso abierto que se distribuye de acuerdo a los términos de la licencia Creative Commons.

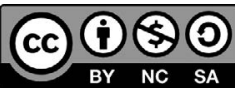

Reconocimiento-

NoComercia-

CompartirIgual 4.0

Internacional

\section{RESUMEN}

El chile Comapeño (C. annuum L.) es un recurso fitogenético nativo de México; sin embargo, la escasez de estudios acerca de su composición química limita su conservación y aprovechamiento sustentables. El objetivo del presente estudio fue evaluar la composición proximal en frutos de tres morfotipos de chile Comapeño y su relación con los factores climáticos. Se determinaron los contenidos de humedad, proteínas, grasas, fibra cruda y cenizas en los frutos deshidratados. Las variables climáticas se obtuvieron de la base de datos de WorldClim. Los datos químicos obtenidos se analizaron mediante estadística descriptiva y se obtuvo el coeficiente de variación. Se llevó a cabo un análisis de correlación de Pearson $(P \leq 0.05)$ para asociar las variables químicas y climáticas. Los frutos de los morfotipos de Veracruz presentaron valores mayores en el contenido de humedad $(>4.11 \%)$, proteínas $(>12.26 \%)$, grasas $(4.26 \%)$ y cenizas $(>6.22 \%)$ que los frutos de Oaxaca; sin embargo, estos últimos presentaron mayor contenido de fibra (12.54\%). Existió correlación significativa ( $|\boldsymbol{r}|>0.70 ; \boldsymbol{P} \leq 0.01)$ entre las variables humedad y climática: temperatura media anual, isotermalidad, intervalo de temperatura anual, precipitación media anual y precipitación del trimestre más seco. La composición proximal de los frutos de tres morfotipos de chile Comapeño de México estuvo asociada con tres variables de temperatura y dos de precipitación. Se recomienda la conservación in situ y ex situ del chile Comapeño, dado que podría contener genes de tolerancia a un determinado factor ambiental.

PALABRAS CLAVE

Caracterización química, variables climáticas, frutos de Capsicum annuum L., análisis de correlación.

\section{ABSTRACT}

The Comapeño chili (C. annuum L.) is a plant genetic resource native of Mexico, but the little research that has been done on its chemical composition limits its conservation and sustainable use. The purpose of the present study was to evaluate the proximal composition in fruits of three Comapeño pepper morphotypes and its relationship with climatic factors. Moisture, protein, fat, crude fiber, and ash content were determined in previously dehydrated fruits. The climatic vari- 
ables were obtained from the WorldClim database. The chemical data obtained were analyzed using descriptive statistics, and the coefficient of variation was obtained. A Pearson correlation analysis $(\mathrm{P} \leq 0.05)$ was carried out to associate the chemical and climatic variables. The fruits of the morphotypes from Veracruz showed higher values in moisture content $(>4.11 \%)$, proteins $(>$ $12.26 \%)$, fats $(4.26 \%)$, and ashes $(>6.22 \%)$ than the fruits from Oaxaca; however, they were higher in fiber (12.54\%). There was a highly significant correlation $(|\boldsymbol{r}|>0.70 ; \boldsymbol{P} \leq 0.01)$ between moisture and climatic variables: annual mean temperature, isothermality, temperature annual range, mean annual precipitation, and precipitation during the driest quarter. The proximal composition in the fruits of the three Comapeño pepper morphotypes from Mexico was associated with three temperature variables and two precipitation variables. Therefore, in situ and ex situ conservation of the Comapeño pepper is recommended since it could contain genes for tolerance to a certain environmental factor.

\section{KEYWORDS}

Chemical characterization, climatic variables, Capsicum annuum L. fruits, correlation analysis.

\section{INTRODUCCIón}

El género Capsicum pertenece a la familia Solanaceae e incluye 25 especies nativas del continente americano (Moscone et al. 2003). México es considerado uno de los centros de origen y domesticación de Capsicum annuum L. (Moscone et al. 2003). Esta especie, que incluye variantes silvestres y cultivadas, se encuentra ampliamente distribuida en toda la República Mexicana, cultivada desde el nivel del mar hasta los 2,500 m (Hernández-Verdugo et al. 2012; Aguirre-Mancilla et al. 2017). La amplitud altitudinal es favorecida por la posición geográfica del país, situado en una zona de transición entre dos regiones biogeográficas, la neártica y neotropical, lo que beneficia la distribución, el endemismo, la riqueza de especies animales y plantas, así como la evolución de las mismas para adaptarse a condiciones ambientales específicas (De Alba y Reyes 1998; CONABIO 2000). Capsicum annumm no es la excepción, debido a su importancia económica y sociocultural.

México produjo 3,238,244.81 $\mathrm{t}$ de frutos de $C$. annuum en tipo verde en 2019, con un valor económico en la producción de $\$ 32,761,548.77$ (SIAP 2020); de ese total, por su importancia económica y social, se destinan 1,053,000.00 $\mathrm{t}$ de fruto al comercio exterior, principalmente a Estados Unidos (SIAP 2018). Además, el consumo nacional per cápita de chile es de $18.1 \mathrm{~kg}$ anual, del cual 75 por ciento es consumido en fruto fresco (González 2010). Los estados de mayor producción de chile verde (C. annuum) que abastecen al mercado consumidor se ubican en el centro y norte del país (Chihuahua, Sinaloa, Zacatecas y San Luis
Potosí), y aportan casi 70 por ciento de la producción nacional (SIAP 2020).

Sin embargo, Chiapas, Oaxaca y Veracruz, ubicados en el sur-sureste, tienen la mayor variedad de morfotipos de $C$. annuum, tanto silvestres como domesticados, lo que se atribuye a la gran riqueza cultural de los pobladores, quienes, a través de los años, le han dado una diversidad de usos (Aguilar-Rincón et al. 2010).

En Oaxaca se han efectuado estudios relacionados con caracterizaciones agromorfológicas en frutos y calidad de semillas en varios morfotipos (López y Pérez 2015; Castellón et al. 2014; Martínez et al. 2019) y fitoquímicas de C. annuum y C. pubescens Ruiz \& Pav., en germoplasma silvestre y domesticado (Vera-Guzmán et al. 2011).

Por su parte, Hernández-Verdugo et al. (2012) analizaron los factores geográficos y climáticos, para concluir que éstos influyen en ciertas características morfológicas como tamaño, peso y número de semillas en frutos de poblaciones silvestres de $C$. annuum var. glabriusculum, del noroeste de México. En Veracruz, Leyva-Ovalle et al. (2018) caracterizaron morfológicamente poblaciones de chile manzano ( $C$. pubescens Ruiz \& Pav.) y encontraron seis grupos poblacionales con características morfológicas diferentes, atribuidas a las condiciones ambientales de los sitios de recolecta.

Es importante mencionar que las investigaciones que involucran evaluaciones químicas en frutos de C. annuum y su relación con factores climáticos son limitadas (Vera-Guzmán et al. 2011; Moreno-Ramírez et al. 2019). No obstante, los estudios anteriores han 
encontrado variación morfológica amplia en planta, flor y calidad química en el fruto de Capsicum spp., y, de esta manera, han propuesto estrategias de conservación y aprovechamiento de los recursos fitogenéticos nativos de México. En este sentido, es relevante explorar, rescatar y caracterizar químicamente la diversidad de los morfotipos de $C$. annuum existente en los huertos familiares con materiales semidomesticados.

En Oaxaca, los agricultores pequeños aún conservan diversos morfotipos de C. annuum, entre ellos el huacle, por su impacto económico alto, pues el fruto se comercializa a un precio de $\$ 250.00 \mathrm{~kg}$ en estado deshidratado (López et al. 2016), además de que los morfotipos nativos tienen un sabor peculiar en las comidas regionales (Aguirre-Mancilla et al. 2017). Por otra parte, el chile Comapeño es sembrado principalmente en huertos familiares en el centro de Veracruz, básicamente para autoconsumo, y es comercializado en fresco y deshidratado de manera local en el mercado (DeWitt y Bosland 2009). Sin embargo, se tienen escasos reportes sobre chile Comapeño, principalmente evaluaciones morfológicas (Jiménez 2018) y uso de sustratos en crecimiento de plantas de esta especie bajo invernadero (Vara 2012), pero las características químicas de los frutos y la relación con los factores climáticos no se ha analizado. Además, se ha ido desplazando a los materiales nativos por la introducción de variedades mejoradas de $C$. annuum, por lo que se requieren esfuerzos para el conocimiento de la variación tanto agromorfológica como química de las poblaciones nativas y su relación con los factores climáticos para proponer estrategias de conservación y aprovechamiento (Allendorf et al. 2012; Hernández-Nicolás et al. 2016). Por lo tanto, el objetivo del presente estudio fue evaluar la composición proximal en frutos de tres morfotipos de chile Comapeño (C. annuum) de Oaxaca y Veracruz, México, y su relación con factores climáticos.

\section{Materiales y Métodos}

\section{Recolección de frutos}

La recolección de los frutos de los morfotipos de chile Comapeño (C. annuum) maduros fisiológicamente para el consumo se hizo de forma manual en huertos familiares durante noviembre y diciembre de 2018. Los frutos fueron seleccionados al azar, libres de daños físicos y patógenos. Posteriormente, las muestras fueron maceradas con un molino eléctrico (Krups GX4100®, México) y conservadas a $-20{ }^{\circ} \mathrm{C}$ hasta su uso en el análisis proximal. Las localidades donde se recolectaron las muestras de chile fueron: Raya Licona, Oaxaca; Xocuapa y Yololtepec, Veracruz (Cuadro 1), y se georreferenciaron con un geoposicionador satelital (GPS) (Garmin eTrex ${ }^{\circledR} 10$ ). Cabe mencionar que, en los lugares muestreados, las personas poseen aún una lengua autóctona (mazateco y náhuatl).

\section{Variables químicas}

La humedad se cuantificó por gravimetría usando desecación en una estufa a $60{ }^{\circ} \mathrm{C}$ con vacío de $60 \mathrm{kPa}$, de acuerdo con el método de AOAC 934.01 (AOAC 1990). El contenido de grasa se extrajo de las muestras deshidratadas usando el método descrito por Soxhlet (1879). Posteriormente, se obtuvo proteína cruda

Cuadro 1. Datos de pasaporte de los morfotipos de C. annuum y la lengua autóctona predominante en las localidades muestreadas.

\begin{tabular}{ccccccc}
\hline $\begin{array}{c}\text { Procedencia del } \\
\text { morfotipo }\end{array}$ & Estado & Municipio & Longitud oeste & $\begin{array}{c}\text { Latitud } \\
\text { norte }\end{array}$ & $\begin{array}{c}\text { Altitud (m) } \\
\text { Lengua } \\
\text { autóctona }\end{array}$ \\
\hline Raya Licona & Oaxaca & $\begin{array}{c}\text { San Miguel } \\
\text { Soyatepec }\end{array}$ & -96.660 & 18.352 & 77 & mazateco \\
Xocuapa & Veracruz & Tezonapa & -96.778 & 18.494 & 311 & 18.616 \\
Yolotepec & Veracruz & Tezonapa & -96.828 & 117 & náhuatl \\
\hline
\end{tabular}


por el método Kjeldahl, de acuerdo con la NOM-F68-S-1980 (SPFI 1980). El contenido de proteína se cuantificó con el contenido de nitrógeno por el factor 6.25 para alimentos (Zaragoza-García et al. 2016). La fibra cruda se analizó usando el método gravimétrico según el AOAC (1990). La ceniza se determinó gravimétricamente por incineración a $550{ }^{\circ} \mathrm{C}$, de acuerdo con el método AOAC 942.05 (AOAC 1990). Los carbohidratos fueron determinados por diferencia de peso del resto de componentes. Todos los análisis se hicieron por triplicado.

\section{Factores climáticos}

Los datos bioclimáticos de cada recolecta de C. annuum se extrajeron de la base de datos WorldClim Global Climate GIS database, mediante el programa de cómputo Diva Gis versión 7.5 (Hijmans et al. 2005) y se muestran en el Cuadro 2.

\section{Análisis de datos}

Los datos de las determinaciones químicas de los morfotipos de chile Comapeño (C. annuum) se analizaron mediante estadística descriptiva $\mathrm{y}$ coeficientes de variación (CV) (Hidalgo 2003). El CV se usó como indicador del grado de variación de las características químicas dentro de cada población o morfotipo (Gutiérrez-Lozano et al. 2020).

Posteriormente, se hizo una selección de variables a partir de 19 bioclimáticas, y sus promedios se utilizaron para construir una matriz de correlaciones, con el objeto de obtener los valores y vectores propios, los cuales, junto con la gráfica de Gabriel (1971), representaron las correlaciones entre variables y su aporte a la variación global (Pla 1986). Con ello, se seleccionaron aquellas variables pares con coeficientes de correlación superior a 0.5 , con alto aporte significativo a la variación climática total, $\mathrm{y}$, sobre todo, que no

Cuadro 2. Variables climáticas utilizadas en la caracterización de los morfotipos de C. annuum de dos estados de México.

\begin{tabular}{|c|c|c|}
\hline Variable (Unidad de medida) & Acrónimo & Fuente \\
\hline Temperatura media anual $\left({ }^{\circ} \mathrm{C}\right)$ & Bio1 & Worldclim \\
\hline Intervalo de las temperaturas medias diurnas $\left({ }^{\circ} \mathrm{C}\right)$ & Bio2 & Worldclim \\
\hline Isotermalidad & Bio3 & Worldclim \\
\hline Estacionalidad de la temperatura (\%) & Bio4 & Worldclim \\
\hline Temperatura máxima del mes más cálido $\left({ }^{\circ} \mathrm{C}\right)$ & Bio5 & Worldclim \\
\hline Temperatura mínima del mes más frío $\left({ }^{\circ} \mathrm{C}\right)$ & Bio6 & Worldclim \\
\hline Intervalo de la temperatura anual (Bio5-Bio6) $\left({ }^{\circ} \mathrm{C}\right)$ & Bio7 & Worldclim \\
\hline Temperatura media del trimestre más lluvioso $\left({ }^{\circ} \mathrm{C}\right)$ & Bio8 & Worldclim \\
\hline Temperatura media del trimestre más seco $\left({ }^{\circ} \mathrm{C}\right)$ & Bio9 & Worldclim \\
\hline Temperatura media del trimestre más cálido $\left({ }^{\circ} \mathrm{C}\right)$ & Bio10 & Worldclim \\
\hline Temperatura media del trimestre más frío $\left({ }^{\circ} \mathrm{C}\right)$ & Bio11 & Worldclim \\
\hline Precipitación media anual (mm) & Bio12 & Worldclim \\
\hline Precipitación del mes más húmedo (mm) & Bio13 & Worldclim \\
\hline Precipitación del mes más seco (mm) & Bio14 & Worldclim \\
\hline Estacionalidad de la precipitación (\%) & Bio15 & Worldclim \\
\hline Precipitación del trimestre más lluvioso (mm) & Bio16 & Worldclim \\
\hline Precipitación del trimestre más seco (mm) & Bio17 & Worldclim \\
\hline Precipitación del trimestre más cálido (mm) & Bio18 & Worldclim \\
\hline Precipitación del trimestre más frío (mm) & Bio19 & Worldclim \\
\hline
\end{tabular}


tuvieran colinealidad. Finalmente, se llevó a cabo el análisis de correlación de Pearson para determinar si existe asociación entre las nueve variables climáticas seleccionadas y las cinco químicas. Dichos análisis se efectuaron mediante el programa Statistical Analysis System, versión 9.1 (SAS Institute 2004).

\section{Resultados y Discusión}

\section{Determinaciones químicas y nivel de variabilidad}

El promedio de humedad de los frutos del morfotipo de C. annuum de Yololtepec (7.01\%) fue diferente al valor de $4.08 \%$ del morfotipo de Raya Licona, el cual tuvo frutos con porcentajes bajos en este parámetro (Cuadro 3). Estos resultados son menores en comparación con los de Solís-Marroquín et al. (2017) y Riquelme y encontrado en el presente trabajo fue superior a los resultados reportados por Giuffrida et al. (2013), quienes mencionaron valores de proteína en los frutos en madurez de consumo en algunas variedades comerciales de Capsicum chinense Jacq., C. annuum y Capsicum frutescens L., entre 9.41 y 12.8 por ciento. Además, estos resultados fueron similares a 12.19 por ciento de proteínas obtenido en los frutos de C. chinense, variedad Jaguar, cultivada en suelos negros de Yucatán (Chel-Guerrero et al. 2020). Estos resultados sugieren, en primer lugar, que las condiciones agroecológicas donde se siembran estos frutos favorecen el desarrollo de proteínas; además, el consumo de los frutos de los morfotipos de Veracruz muestra viabilidad como complemento nutricional en la dieta humana, considerando que la ingesta de proteína recomendada en un adulto sano va de 1 a $1.5 \mathrm{~g} / \mathrm{kg} /$ día (Bonet et al. 2011). Es relevante obtener el valor nutricional de las especies de plantas nativas para proponer estrategias

Cuadro 3. Análisis proximal en tres morfotipos de chile comapeño (C. annuum) nativos de Oaxaca y Veracruz, México (g/100 g muestra húmeda).

\begin{tabular}{|c|c|c|c|c|c|}
\hline Procedencia del morfotipo & Humedad (\%) & Proteínas (\%) & $\begin{array}{c}\text { Grasas } \\
(\%)\end{array}$ & Fibra cruda $(\%)$ & $\begin{array}{c}\text { Cenizas } \\
(\%)\end{array}$ \\
\hline Raya Licona & $4.09 \pm 0.009$ & $8.70 \pm 1.56$ & $3.87 \pm 0.55$ & $12.54 \pm 0.03$ & $5.97 \pm 0.001$ \\
\hline Xocuapa & $4.11 \pm 0.03$ & $12.79 \pm 0.99$ & $4.37 \pm 0.12$ & $12.41 \pm 0.26$ & $6.71 \pm 1.0$ \\
\hline Yololtepec & $7.01 \pm 0.28$ & $12.26 \pm 1.38$ & $4.26 \pm 0.33$ & $11.96 \pm 0.24$ & $6.22 \pm 0.22$ \\
\hline Promedio & 5.07 & 11.25 & 4.17 & 12.30 & 6.31 \\
\hline $\mathrm{CV}^{*}$ & 34.0 & 26.0 & 7.0 & 2.5 & 6.0 \\
\hline
\end{tabular}

Cada valor es el promedio de tres replicas $\pm \mathrm{DE} .{ }^{*} \mathrm{DE}=$ Desviación estándar, ${ }^{*} \mathrm{CV}=$ Coeficiente de variación.

Matiacevich (2017), quienes registraron 85.8 y 84.1 por ciento de humedad en frutos en madurez de consumo (rojo) de C. annuum var. 'Siete Caldos' y 'Cacho de Cabra', respectivamente. No obstante, el promedio de 7.01 por ciento de Yololtepec se aproximó a los reportados por Orellana-Escobedo et al. (2013), quienes obtuvieron contenidos de humedad en un intervalo de 5.4 a 13.0 por ciento en frutos de diferentes tipos de chile de la región noroeste de Chihuahua, México.

Los frutos del morfotipo de Yololtepec obtuvieron el mayor contenido de proteína $(12.8 \%)$, en contraste con el de Raya Licona (8.70\%), posiblemente debido al contraste de temperatura media anual entre ambos sitios: en el primero, $22.1{ }^{\circ} \mathrm{C}$ y en el segundo, $26{ }^{\circ} \mathrm{C}$. El valor promedio de 12.8 por ciento de proteína de conservación y aprovechamiento sustentable (Allendorf et al. 2012), con la finalidad de identificar un morfotipo de chile Comapeño potencial que cubra los requerimientos nutricionales, de acuerdo con las necesidades de los mercados consumidores.

El morfotipo de Xocuapa tuvo frutos con el mayor contenido de grasas (4.37\%), y las muestras de Raya Licona correspondieron a frutos con menor porcentaje (3.87\%) (Cuadro 3). Estos resultados están dentro de los intervalos observados por Solís-Marroquín et al. (2017), quienes obtuvieron entre 4.50 y 8.0 por ciento de grasa en frutos de la var. 'Siete Caldos' (C. annuum), con diferentes estadíos de madurez comercial. En contraste, Riquelme y Matiacevich (2017) encontraron 0.9 por ciento de grasa en muestras humedas de frutos 
de chile var. 'Cacho de Cabra', lo que evidencia que dicho valor está por debajo de lo que contienen los morfotipos de chile Comapeño.

Los frutos del morfotipo Raya Licona tuvieron el porcentaje de fibra más alto (12.53\%) en comparación con los frutos de los lugares de Xocuapa y Yololtepec (12.41 y 11.96\%, respectivamente) (Cuadro 3). Los valores de los morfotipos de Raya Licona y Xocuapa son comparables al obtenido con la var. 'Siete Caldos' (C. аппиит), en el cual se reportaron promedios de 12.0 y 12.5 por ciento de fibra cruda en frutos de color rojo y rojo-quemado-oscuro, evaluados en condiciones de cielo abierto (Solís-Marroquín et al. 2017). Asimismo, los niveles de fibra cruda registrados en el germoplasma Comapeño fueron similares a lo encontrado por Chel-Guerrero et al. (2020), al estudiar los frutos de $C$. chinense, cultivado en suelos negro y rojo de Yucatán, con valores de fibra de 12.62 y 13.20 por ciento, respectivamente.

El morfotipo de C. annuum del sitio de Xocuapa presentó el contenido de cenizas totales más alto $(6.71 \%)$ y el de Raya Licona registró el valor menor (5.97\%) (Cuadro 3). En el presente estudio, los niveles de cenizas son similares a los valores de 5.63 a 6.5 por ciento encontrados en frutos de la variedad Siete Caldos (C. annuum), bajo condiciones de cielo abierto (Solís-Marroquín et al. 2017).

Con base en los resultados obtenidos, se pudo observar que los morfotipos de Veracruz respecto a los de Oaxaca presentaron los promedios de humedad, proteínas, grasas y cenizas más altos, posiblemente porque el chile Comapeño, además de ser nativo de la zona centro del estado de Veracruz (DeWitt y Bosland 2009), es conservado en los huertos familiares por la cultura náhuatl para fines de autoconsumo y comercialización en el mercado local. En cambio, la cultura mazateca lo destina sólo para el autoconsumo. Aunado a ello, los agricultores han desempeñado un papel muy importante en la diferenciación de los morfotipos de $C$. annuum, mediante el manejo, selección e intercambio de material genético.

Las características de humedad y proteínas a nivel intramorfotipos de chile Comapeño, procedentes de Oaxaca y Veracruz, presentaron un coeficiente de variación $(\mathrm{CV})$ de 34 y 26 por ciento, respectivamente (Cuadro 3). Dado que el CV indica la magnitud de la variabilidad de las características dentro de un germoplasma determinado (Hidalgo 2003), los resultados en el presente estudio indicaron una variabilidad química amplia entre los morfotipos de C. annuum. Si bien el grado de variación de una característica no revela necesariamente la utilidad en un programa de aprovechamiento, dicha variación puede estar influida por el ambiente y tamaño de muestra analizada, tal como Do Rêgo et al. (2011) enfatizan en su estudio morfológico y químico en frutos de Capsicum sp.

\section{Correlaciones químicas y climáticas}

Primeramente, se llevó a cabo el proceso de selección de variables climáticas, en el cual mostraron colinealidad las bio4, bio5, bio6, bio8, bio9, bio10, bio11, bio15, bio14 y bio19, por lo que se descartaron del análisis de correlación. Los valores de correlación entre variables químicas y climáticas en tres morfotipos de chile Comapeño (C. annuum) se muestran en el Cuadro 4 , en donde se encontró correlación significativa

Cuadro 4. Coeficientes de correlaciones entre variables químicas y climáticas en tres morfotipos de C. annuum de México.

\begin{tabular}{|c|c|c|c|c|c|c|c|c|c|c|c|c|c|c|}
\hline & $\begin{array}{l}\% \\
\mathbf{P}\end{array}$ & $\begin{array}{l}\% \\
G\end{array}$ & $\begin{array}{l}\% \\
\mathrm{~F}\end{array}$ & $\begin{array}{l}\% \\
\mathrm{C}\end{array}$ & $\begin{array}{l}\% \\
\mathrm{H} \\
\end{array}$ & Bio1 & Bio2 & Bio3 & Bio7 & Bio12 & Bio13 & Bio16 & Bio17 & Bio18 \\
\hline$\% \mathrm{P}$ & & 0.62 & -0.28 & 0.33 & 0.38 & -0.42 & $0.73^{*}$ & 0.68 & 0.07 & 0.05 & -0.45 & -0.35 & 0.34 & -0.66 \\
\hline$\%$ G & & & -0.50 & 0.39 & 0.15 & -0.23 & 0.55 & 0.43 & 0.12 & -0.10 & -0.38 & -0.31 & 0.18 & -0.51 \\
\hline$\% \mathrm{~F}$ & & & & -0.24 & $-0.79^{*}$ & $0.85^{*}$ & -0.00 & $-0.82^{*}$ & 0.65 & -0.66 & -0.35 & -0.44 & $-0.83^{*}$ & -0.09 \\
\hline$\% \mathrm{C}$ & & & & & -0.09 & 0.06 & 0.59 & 0.16 & 0.36 & -0.35 & -0.53 & -0.49 & -0.11 & -0.58 \\
\hline$\% \mathrm{H}$ & & & & & & $-0.99^{* *}$ & -0.19 & $0.89^{* *}$ & $-0.88^{* *}$ & $0.88^{* *}$ & 0.58 & 0.68 & $0.90^{* *}$ & 0.30 \\
\hline
\end{tabular}

Nivel de significancia de la correlación: *P $\leq 0.05,{ }^{* *} \mathrm{P} \leq 0.01,{ }^{*}=$ significante, ${ }^{* *}$ altamente significante. Porcentaje de $\mathrm{P}=$ proteína, $\mathrm{G}=$ grasas, $\mathrm{F}=$ fibra, $\mathrm{C}=$ cenizas, $\mathrm{H}=$ humedad. Los acrónimos de las bio1-bio18 se presentan en el Cuadro 2. 
$(\mathrm{P} \leq 0.05)$ entre los niveles de proteína del fruto y la Bio2 (intervalo de las temperaturas medias diurnas). Además, correlaciones significativas $(\mathrm{P} \leq 0.05)$ fueron observadas entre los porcentajes de fibra cruda y las bio1 (temperatura media anual), bio3 (isotermalidad) y bio17 (precipitación del trimestre más seco).

Asimismo, existieron correlaciones altamente significativas ( $\mathrm{P} \leq 0.01)$ entre las variables de humedad y las bio1 (temperatura media anual), bio3 (isotermalidad), bio7 (intervalo de la temperatura anual), bio12 (precipitación media anual) y bio17 (precipitación del trimestre más seco). No obstante, los niveles de grasas y cenizas no evidenciaron correlación significativa con las variables climáticas analizadas; por tanto, más adelante no se dan detalles de estas asociaciones. La clasificación de las correlaciones presentada en este estudio fue acorde con los criterios establecidos en el trabajo de Ruiz-De Anda et al. (2019).

El contenido de proteínas mostró correlación moderada con el contenido de grasas (0.62), la bio3 (isotermalidad) (0.68), y correlación negativa con la bio18 (precipitación del trimestre más cálido) $(-0.66)$; en tanto que ostentó relación alta con la bio2 (intervalo de las temperaturas medias diurnas) (0.73). El nivel de fibra cruda se correlacionó altamente con el porcentaje de humedad (-0.79), la bio1 (temperatura media anual) (0.85), la bio3 (isotermalidad) (-0.82) y la bio17 (precipitación del trimestre más seco) (-0.83). Asimismo, tuvo una asociación moderada con bio7 (intervalo de la temperatura anual) (0.65) y bio12 (precipitación media anual) (-0.66).

El contenido de humedad mostró correlación negativa alta con la bio1 (temperatura media anual) (-0.99) y la bio7 (intervalo de la temperatura anual) $(-0.88)$. No obstante, obtuvo una correlación positiva alta con las bio3 (isotermalidad) (0.89), bio12 (precipitación media anual) (0.88) y bio17 (precipitación del trimestre más seco) (0.90).

Las correlaciones altas ( $|r|>0.70 ; P \leq 0.01)$ entre las características químicas y climáticas citadas en el presente estudio permiten postular que las variables de precipitación y temperatura del lugar de recolecta influyen en las de química proximal (contenido de proteína, fibra y humedad) de los frutos de los morfotipos de chile Comapeño. En ese tenor, Gepts (2008) enfatiza que las variables climáticas relevantes en los procesos fisiológicos y de desarrollo de las plantas son la radiación solar, temperatura media del aire, temperatura y precipitación media anual. Amador et al. (2014) determinaron que el crecimiento de órganos reproductivos (yemas, flores y frutos) y el rendimiento del chile mirasol (C. annuum) fueron influidos por las condiciones climáticas del estado de Zacatecas. Por su parte, Hernández-Verdugo et al. (2012) encontraron que las características morfológicas de tamaño, peso y número de semillas en frutos de poblaciones silvestres de C. annuum var. Glabriusculum están correlacionadas con la precipitación y temperatura media anual, lo que indica alta dependencia con el lugar de origen de las recolectas. Por otra parte, Moreno-Ramírez et al. (2019), mediante Sistemas de Información Geográfica, no encontraron una asociación entre la variabilidad fitoquímica (actividades de eliminación de radicales libres) y el lugar de origen de poblaciones silvestres de chile piquín (C. annuum var. Glabriusculum); en este sentido, la variación puede deberse a otros factores, como el flujo genético entre las poblaciones.

Es relevante destacar que los frutos de chile Comapeño mostraron altos contenidos de proteína y fibra cruda, los cuales permiten mejorar los procesos de digestión en el intestino delgado del ser humano; asimismo, el valor elevado de cenizas de los frutos puede estar relacionado con contenido importante de macro y micro nutrientes, con los cuales se podrían llevar a cabo investigaciones que permitan determinar los elementos relacionados con aspectos nutrimentales y contaminación. En cuanto al almacenamiento para su comercialización, los frutos analizados en el presente estudio presentaron bajos contenidos de humedad, lo que les confiere la calidad suficiente para mantenerse libres de enfermedades en condiciones de poca luz (Solís-Marroquín et al. 2017).

\section{Conclusiones}

Se encontró composición química significativa en los frutos de tres morfotipos de chile Comapeño (C. annuum) nativos de Veracruz y Oaxaca, en las características de proteínas y humedad. Los valores de humedad, proteínas, grasas y cenizas más altos se obtuvieron en los morfotipos de Veracruz respecto a los frutos de Oaxaca. Se encontró correlación altamente significativa entre tres características químicas de los frutos y las variables climáticas en 
tres morfotipos de chile Comapeño (C. annuum) evaluados, lo que sugiere asociación significativa con dos de precipitación y tres de temperatura. Derivado de los resultados anteriores, es importante conservar el chile Comapeño, debido a que puede contener genes tolerantes a cierto factor ambiental. 


\section{Literatura Citada}

Aguilar-Rincón VH, Corona-Torres TP, López-López L, Latournerie-Moreno M, Ramírez-Meraz M, VillalónMendoza H, Aguilar-Castillo JA. 2010. Los chiles de México y su distribución. Colegio de Postgraduados/ SINAREFI/INIFAP/IT-Conkal/UANL/UAN. Montecillo, México.

Aguirre-Mancilla CL, Iturriaga de la Fuente G, RamírezPimentel JG, Covarrubias-Prieto J, Chablé-Moreno F, Raya-Pérez JC. 2017. El chile (C. annuum L.), cultivo y producción de semilla. Ciencia y Tecnología Agropecuaria de México 5: 19-27.

Allendorf FW, Luikart GH, Aitken SN. 2012. Conservation and the Genetics of Populations. Wiley-Blackwell. Chichester, UK.

Amador MD, Velásquez R, Sánchez BI, Acosta E. 2014. Floración y fructificación de chile mirasol (Capsicum annuum L.) con labranza reducida, labranza convencional o incorporación de avena al suelo. Revista Mexicana de Ciencias Agrícolas 6: 1001-1013. https:// doi.org/10.29312/remexca.v5i6.885

[AOAC] Association of Official Analytical Chemists. 1990. Official Methods of Analysis. Association of Official Analytical Chemists. Washington, USA.

Bonet A, Márquez JA, Serón C. 2011. Recomendaciones para el soporte nutricional y metabólico especializado del paciente crítico. Actualización. Consenso SEMICYUC-SENPE: requerimientos de macronutrientes y micronutrientes. Medicina Intensiva 35(Supl. 1): 17-21. https://doi.org/10.1016/S0210-5691(11)70004-3

Castellón E, Carrillo-Rodríguez JC, Chávez-Servia JL, Vera-Guzmán AM. 2014. Variación fenotípica de morfotipos de chile (Capsicum annuum L.) nativo de Oaxaca, México. Pyton 83: 225-236.

Chel-Guerrero LD, Ruíz MC, Rodríguez IM, Ramírez MO, Ramírez EJ. 2020. Evaluación química y uso potencial de subproductos de Capsicum chinense Jacq., cultivado en dos tipos de suelo de Yucatán. En: Rodríguez IM, Ramírez MO, Ramírez EJ, editores. Metabolómica y cultivo del chile habanero (Capsicum chinense Jacq) de la Península de Yucatán. Guadalajara, Centro de Investigación y Asistencia en Tecnología y Diseño del Estado de Jalisco A. C. P. 192-216.

[CONABIO] Comisión Nacional para el Conocimiento y Uso de la Biodiversidad. 2000. Estrategia Nacional sobre Biodiversidad de México. Comisión Nacional para el Conocimiento y Uso de la Biodiversidad. D. F., México.
De Alba E, Reyes ME. 1998. Contexto físico. En: Comisión Nacional para el Conocimiento y Uso de la Biodiversidad, editor. La diversidad biológica de México: Estudio de País, 1998. D. F., Comisión Nacional para el Conocimiento y Uso de la Biodiversidad. P. 4-22.

DeWitt D, Bosland PW. 2009. The Complete Chile Pepper Book: A Gardener's Guide to Choosing, Growing, Preserving, and Cooking. Timber Press, Inc. Portland, USA.

Do Rêgo ER, Do Rego MM, De Matos IWF, Barbosa LA. 2011. Morphological and chemical characterization of fruits of Capsicum spp. accessions. Horticultura Brasileira 29: 364-371.

Gabriel KR. 1971. The biplot graphic display of matrices with application to principal component analysis. Biometrika 58: 453-467. https://doi.org/10.1093/ biomet/58.3.453

Gepts P. 2008. Tropical environments, biodiversity, and the origin of crops. En: Moore P, Ming R, editores. Genomics of Tropical Crop Plants. Nueva York, Springer. P. 1-20.

Giuffrida D, Dugo P, Torre G, Bignardi C, Cavazza A, Corradini C, Dugo G. 2013. Characterization of 12 Capsicum varieties by evaluation of their carotenoid profile and pungency determination. Food Chemistry 140: 794-802. https://doi.org/10.1016/j. foodchem.2012.09.060

González JA. 2010. El cultivo del chile Capsicum annuum L. Boletín.SecretaríadeAgricultura,Ganadería,Desarrollo Rural, Pesca y Alimentación. Guanajuato, México. Gutiérrez-Lozano M, Sánchez-González A, VázquezGarcía JA, López-Mata L, Octavio-Aguilar P. 2020. Diferenciación morfológica poblacional de Magnolia rzedowskiana (Magnoliaceae): especie endémica en peligro de extinción de la Sierra Madre Oriental, México. Revista Mexicana de Biodiversidad 91:e913101. https://doi.org/10.22201/ib.20078706e.2020.91.3101

Hernández-Nicolás NY, Córdova-Téllez L, Luna-Cavazos M, Romero-Manzanares A, Jiménez-Ramírez J. 2016. Morphological variation related with environmental factors in endemic and threatened Jatropha species of Tehuacan-Cuicatlan, Mexico. Genetic Resources and Crop Evolution 64: 557-568. https://doi.org/10.1007/ s10722-016-0383-2

Hernández-Verdugo S, Dávila A, Oyama K. 1999. Síntesis del conocimiento taxonómico, origen y domesticación 
del género Capsicum. Boletín de la Sociedad Botánica de México 64: 65-84.

Hernández-Verdugo S, Porras F, Pacheco-Olvera A, López-España RG, Villarreal-Romero M, Parra-Terraza S, Osuna T. 2012. Caracterización y variación ecogeográfica de poblaciones de chile (Capsicum annuum var. Glabriusculum) silvestre del noroeste de México. Polibotánica 33: 175-191.

Hidalgo R. 2003. Variabilidad genética y caracterización de especies vegetales. En: Franco TL, Hidalgo R, editores. Análisis estadístico de datos de caracterización morfológica de recursos fitogenéticos, vol. 8. Colombia, IPGRI. P. 2-26.

Hijmans RJ, Cameron SE, Parra JL, Jones PG, Jarvis A. 2005. Very high resolution interpolated climate surfaces for global land areas. International Journal of Climatology 25: 1965-1978. https://doi.org/10.1002/joc.1276

Jiménez BG. 2018. Caracterización morfológica de morfotipos de chile Comapeño (Capsicum annuum L.) de la región de Tezonapa, Veracruz. Tesis de Licenciatura. Instituto Tecnológico Superior de Zongolica. Veracruz, México.

Leyva-Ovalle OR, Andrés-Meza P, Del Valle-Hernández D, Meneses-Márquez I, Murguía-González J, GalindoTovar ME, López-Sánchez H, Serna-Lagunes R, Del Rosario-Arellano L, Lee-Espinoza HE, Sierra-Macías M, Espinoza-Calderón A. 2018. Morphological characterization of manzano hot pepper (Capsicum pubescens Ruiz \& Pav.) landraces in the central region of Veracruz state, Mexico. Revista Bio Ciencias 5: e388. https://doi. org/10.15741/revbio.05.e388

López P, Pérez D. 2015. El chile Huacle (Capsicum annuum sp.) en el estado de Oaxaca, México. Agroproductividad 8: 35-38.

López P, Rodríguez R, Bravo E. 2016. Impacto económico del chile Huacle (Capsicum annuum L.) en el estado de Oaxaca. Revista Mexicana de Agronegocios 38: 317-328. https://doi.org/10.22004/ag.econ.239292

Martínez JSS, Aquino-Bolaños T, Donají-Hernández Y, Cruz-Izquierdo S. 2019. Características de fruto y semilla de chile huacle (Capsicum annuum L.) producido en hidroponía. IDESIA (Chile) 37: 87-94.

Moreno-Ramírez YdelR, Hernández-Bautista A, López PA, Vanoye-Eligio V, Torres-Rodríguez ML, Torres-Castillo JA. 2019. Variability in the phytochemical contents and free radical-scavenging capacity of Capsicum annuum var. glabriusculum (wild Piquin Chili). Chemistry
\& Biodiversity 16: e1900381. https://doi.org/10.1002/ cbdv.201900381

Moscone EA, Baranyi M, Ebert I, Greilhuber J, Ehrenorfer F, Hunzijer AT. 2003. Analysis of nuclear DNA content in Capsicum (Solanaceae) by flow cytometry and Feulgen densitometry. Annals Botany 92: 21-29.

Orellana-Escobedo L, Garcia-Amezquita LE, Olivas GI, Ornelas-Paz JJ, Sepúlveda DR. 2013. Capsaicinoids content and proximate composition of Mexican chili peppers (Capsicum spp.) cultivated in the State of Chihuahua. CyTA-Journal of Food 11: 179-184.

Pla LE. 1986. Análisis multivariado: método de componentes principales. Programa Regional de Desarrollo Científico y Tecnológico/Organización de Estados Americanos. Washington, USA.

Riquelme N, Matiacevich S. 2017. Characterization and evaluation of some properties of oleoresin from Capsicum annuum var. cacho de cabra. CyTA - Journal of Food 15: 344-351. https://doi.org/10.1080/19476337.2 016.1256913

Ruiz-De Anda D, Ventura-Lara MG, Rodríguez-Hernández G, Ozuna C. 2019. The impact of power ultrasound application on physicochemical, antioxidant, and microbiological properties of fresh ornage and celery juice blend. Journal of Food Measurement and Characterization 13: 3140-3148. https://doi.org/10.1007/ s11694-019-00236-y

[SAS] SAS Institute. 2004. SAS/STAT, 9.1. User's Guide. SAS Institute Inc. Cary, USA.

[SIAP] Servicio de Información Agroalimentaria y Pesquera. 2018. Atlas Agroalimentario 2012-2018. SAGARPA. Ciudad de México, México.

[SIAP] Servicio de Información Agroalimentaria y Pesquera. [internet]. 2020. Cierre de la producción agrícola por cultivo. Anuario Estadístico de la Producción Agrícola. [citado 2020 Abril 01]. Disponible en: https://nube.siap. gob.mx/cierreagricola/

[SPFI] Secretaría de Patrimonio y Fomento Industrial. 1980. NOM-F-68-S-1980. Alimentos Determinación de Proteínas. Diario Oficial de la Federación del 4 de agosto de 1980.

Solís-Marroquín D, Lecona-Guzmán CA, Ruiz-Lau N, Ocampo P, Rodas-Trejo J, Gonzales-Santiago C, González-Mejía O, Gordillo-Páez L. 2017. Análisis bromatológico de frutos de chile "siete caldos" (Capsicum annuиm) cultivados en condiciones de cielo abierto y casa sombra. Agroproductividad 9: 34-40. 
Soxhlet F. 1879. Die gewichts-analytische Bestimmung des Milchfettes. Dinglers Polytechnisches Journal 232: 461.

Vara MJC. 2012. Crecimiento y desarrollo del chile habanero (Capsicum chinense Jacq.) y chile Comapeño (Capsicum annuum L.) en tres diferentes sustratos, bajo condiciones de agricultura protegida. Trabajo de experiencia recepcional. Universidad Veracruzana. Xalapa, México.

Vera-Guzmán AM, Chávez-Servia JL, Carrillo-Rodríguez JC, López MG. 2011. Phytochemical evaluation of wild and cultivated pepper (Capsicum annuum L. and C. pubescens Ruiz \& Pav.) from Oaxaca, México. Chilean Journal of Agricultural Research 71: 578-585.

Zaragoza-García JM, García-López IG, Guzmán-Mar JL. 2016. Validación del método para la determinación de proteína en alimentos bajo la norma NMX-F-608NORMEX-2011. Investigación y Desarrollo en Ciencia y Tecnología de Alimentos 1: 863-869. 